\title{
Polarization measurements of Gamma-Ray Bursts with IBIS
}

\section{Diego Götz ${ }^{* \dagger}$}

CEA Saclay - DSM/Irfu/SAp - AIM

Orme des Merisiers, Bât. 709, F-91191, Gif-sur-Yvette Cedex, France

E-mail: diego.gotz@cea.fr

\section{Philippe Laurent}

CEA Saclay - DSM/Irfu/SAp - APC

10, rue Alice Domon et Léonie Duquet, F-75205, Paris Cedex 13, France

E-mail: plaurent@cea.fr

\section{François Lebrun}

CEA Saclay - DSM/Irfu/SAp - APC

10, rue Alice Domon et Léonie Duquet, F-75205, Paris Cedex 13, France

E-mail: flebrun@cea.fr

The measurement of polarization is a powerful tool to constrain physical processes in astrophysical sources. Here we present the results of the measurements of linear polarization obtained in the 200-800 keV energy band using IBIS as a Compton polarimeter. We will focus on the highly polarized GRB 041219A, and its time resolved analysis. For this GRB our results favor models where the prompt emission at these energies is produced by synchrotron radiation from a relativistic outflow with a magnetic field, which is coherent on an angular size comparable with the angular size of the emitting region. Finally, we present the measurements and upper limits obtained for other GRBs detected by INTEGRAL.

8th INTEGRAL Workshop "The Restless Gamma-ray Universe"

September 27-30 2010

Dublin Castle, Dublin, Ireland

\footnotetext{
*Speaker.

${ }^{\dagger}$ This work was supported by the Centre National d'Etudes Spatiales (CNES). It is based on observations with IBIS embarked on INTEGRAL, an ESA project with instruments and science data centre funded by ESA member states, Poland and with the participation of Russia and the USA.
} 


\section{Introduction}

Despite the recent advances in Gamma-Ray Bursts (GRBs) science [1], there is no general consensus on the nature of the prompt gamma-ray emission. While some models favor purely synchrotron emission others include inverse Compton processes in order to explain the $\mathrm{keV}$ to $\mathrm{GeV}$ emission recently observed by Fermi[2]. On the other hand, the idea of a relativistic outflow being at the origin of the prompt GRB phenomenon is widely accepted, but its geometrical structure, as well as the energy dissipation mechanisms - internal shocks[3] or magnetic dissipation[4] - are still debated. The measure of the polarization of GRB radiation is a powerful tool to investigate the radiative processes of the emitting region as well as its geometrical structure.

\section{GRB 041219A}

Thanks to its two superposed detector layers, ISGRI and PICsIT, IBIS[5] on board INTEGRAL can be used as a Compton telescope. This mode is sensitive to the linear polarization of radiation, and has allowed us to measure the polarization fraction, $\Pi$, and angle, $P A$, for the Crab nebula[6] and for GRB 041219A[7]. The results on GRB 041219 are reported on Figs. 1, 2 and 3, and Table 1 , and show that while integrating over the entire duration of the GRB, no significant polarization can be measured, a time resolved analysis showed that the polarization level is significant (e.g. the chance probability during P8 is of the order of $10^{-4}$ ) and its intensity and angle are highly variable over the event, reaching fairly high intensities. For details on the analysis, see [7]. In Fig. 3 we present the contour plots of the error estimation of the polarization factor level and angle, following the Eq. 2 from [6]. One can see that for most intervals, especially the second peak, P6, P8 or P30, the hypothesis of an unpolarized source can be excluded at a confidence larger than $99 \%$.

\begin{tabular}{ccccc}
\hline Name & $\mathrm{T}_{\text {start }}$ & $\mathrm{T}_{\text {stop }}$ & $\Pi$ & $P A$ \\
& U.T. & U.T. & $\%$ & degrees \\
\hline First Peak & $01: 46: 22$ & $01: 47: 40$ & $<4$ & - \\
Second Peak & $01: 48: 12$ & $01: 48: 50$ & $43 \pm 25$ & $38 \pm 16$ \\
P6 & $01: 46: 47$ & $01: 46: 57$ & $22 \pm 13$ & $121 \pm 17$ \\
P8 & $01: 46: 57$ & $01: 47: 07$ & $65 \pm 26$ & $88 \pm 12$ \\
P28 & $01: 48: 37$ & $01: 48: 47$ & $42 \pm 42$ & $106 \pm 37$ \\
P30 & $01: 48: 47$ & $01: 48: 57$ & $90 \pm 36$ & $54 \pm 11$ \\
\hline PP1 & $01: 46: 47$ & $01: 47: 12$ & $<13$ & - \\
PP2 & $01: 48: 32$ & $01: 48: 57$ & $54 \pm 24$ & $42 \pm 13$ \\
\hline
\end{tabular}

Table 1: Polarization results for the different time intervals. Errors are given at $1 \sigma$ c.l. for one parameter of interest.

Although the statistical errors on these measurements remain rather high, our $200-800 \mathrm{keV}$ data favor models involving synchrotron radiation from a relativistic outflow with an ordered magnetic field (in a region of angluar size $\sim 1 / \Gamma$ ) as it seems to have the best capabilities to maintain a high polarization level with some variability. This geometry is favored if the field is carried by the outflow from the central source, as the poloidal component decreases much faster with radius than 


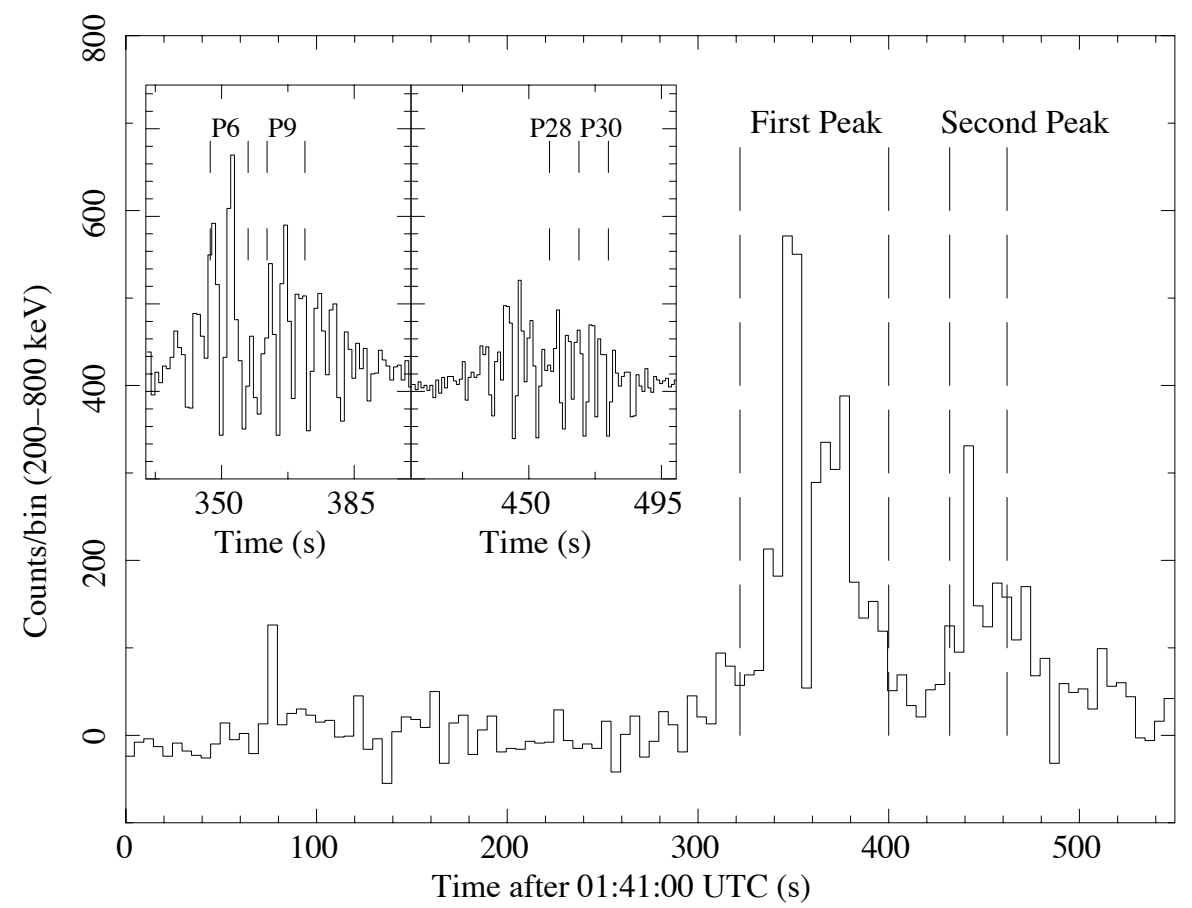

Figure 1: Compton mode light curve of GRB 041219A. From [7].

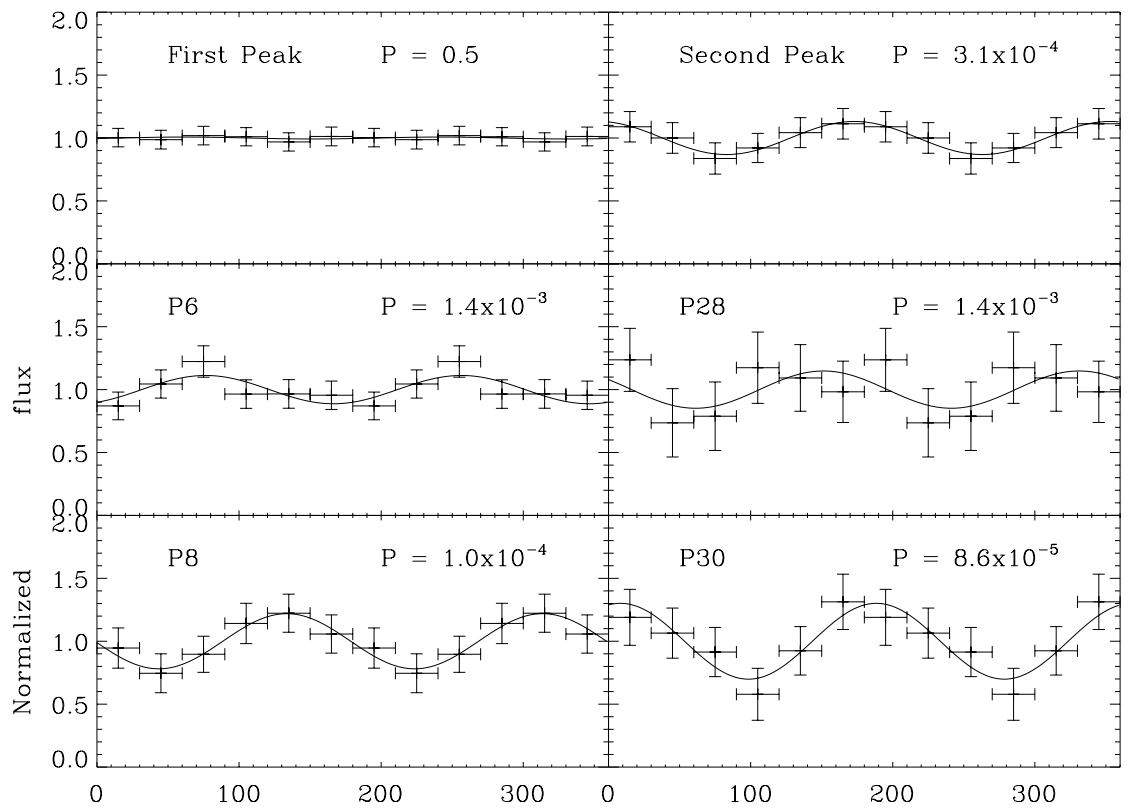

Figure 2: Polarigrams (i.e. flux vs scattering angle in degrees) of the different phases of the GRB. From [7] 

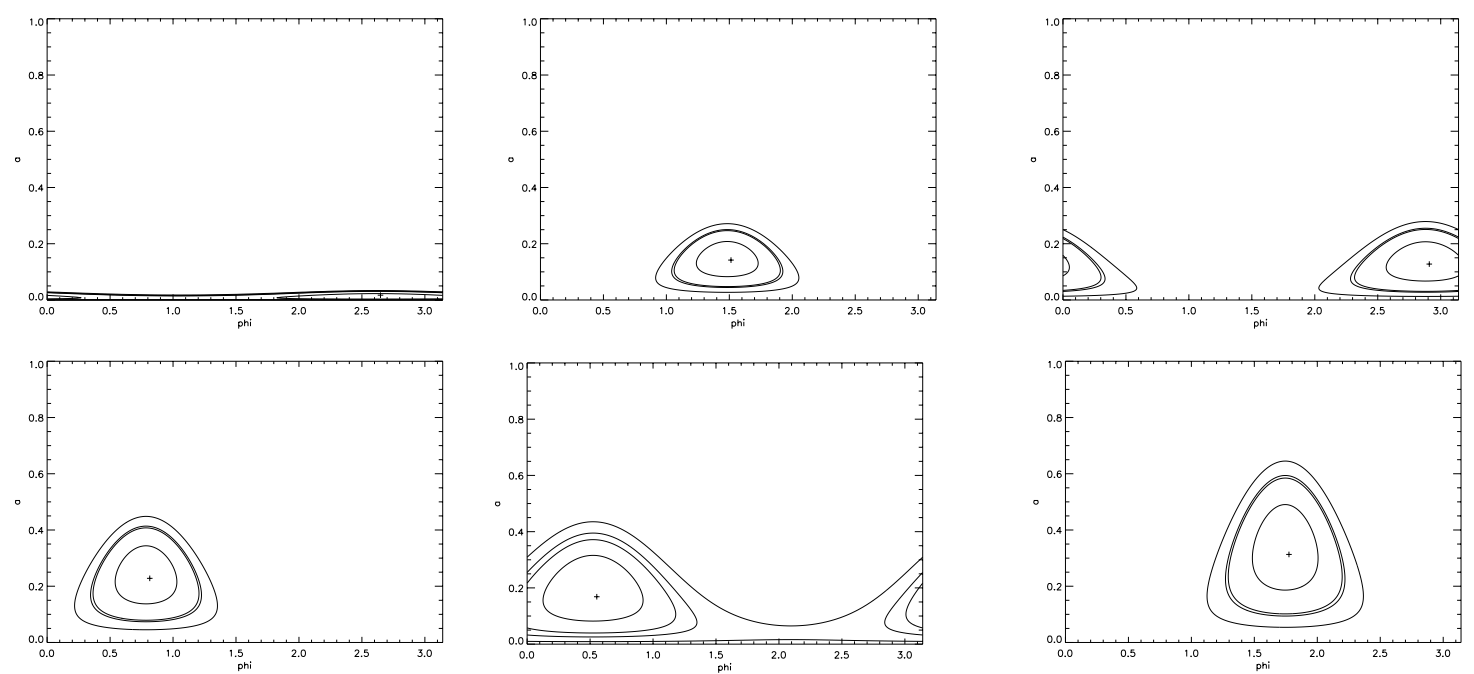

Figure 3: Contour plots of the relative polarization level ( $a$; $\Pi$ is given by $a / a_{0}$, where $a_{0}$ is about 0.3 ) versus the polarization angle ( $p h i$ in radians; $P A=$ phi $-\pi / 2+n \pi$ ) for the same time intervals as in Fig. 2 . From left to right and from top to bottom: first peak, second peak, P6, P8, P28, P30. The confidence levels are at $67,90,95$, and $99 \%$.

the toroidal one. The polarization level at the peak of a given pulse can be as high as $\Pi / \Pi_{\mathrm{yn}} \sim 0.8$, i.e. $\Pi \sim 60 \%$, leading to a maximum time-averaged polarization in long intervals of $\Pi / \Pi_{\max } \sim 0.6$, i.e. $\Pi \sim 45 \%$ in this case $[9,10,8]$. The main requirement is to have a uniform magnetic field in space, i.e. with a coherent scale $R \theta_{\mathrm{B}}$ with $\theta_{\mathrm{B}} \gtrsim 1 / \Gamma$. The fact that the polarization level and angle vary during the burst indicates on the other hand that the field is not necessarily uniform in time, as it would be required to explain a high level of the time-integrated polarization [8]. A magnetic field anchored in the central engine and carried by the outflow to large distance [11] is therefore not the only possibility. A magnetic field generated at the shock could also work, and even favor variability, if there is a process capable to increase the field coherence scale (the field is most probably initially generated on small, skin-depth, scales). The existence of such a process is unclear in our present knowledge of the micro-physics in mildly relativistic shocks. Note that the condition $\theta_{\mathrm{B}} \gtrsim 1 / \Gamma$ is really necessary only in the pulses with the highest level of polarization. If $\theta_{\mathrm{B}}$ is smaller, so that a number $N \sim\left(\Gamma \theta_{\mathrm{B}}\right)^{-2}$ of mutually incoherent patches are present in the visible region, the level of polarization will decrease, but the variability (both of the polarization level and angle) will increase [9]. If the radiating electrons are accelerated in internal shocks $[3,12,13]$, the Lorentz factor is necessarily varying in the outflow, which can be an additional source of variability for the polarization. If $\theta_{\mathrm{B}}$ and $1 / \Gamma$ are close, the number of coherent patches in the visible region could vary from a pulse to another. We therefore conclude that any scenario where the observed gamma-rays are produced by synchrotron radiation from electrons in a relativistic jet with an ordered magnetic field in the plane perpendicular to the jet velocity seems fully consistent with our observations, as long as the coherence scale $\theta_{\mathrm{B}}$ of the field is larger than $1 / \Gamma$ in most of the emitting regions. A potential difficulty remains: an additional random component of the magnetic field is probably necessary to allow for particle acceleration in shocks. This component would reduce the level of polarization by a factor that is however difficult to estimate, as the intensity of the former is not well constrained 

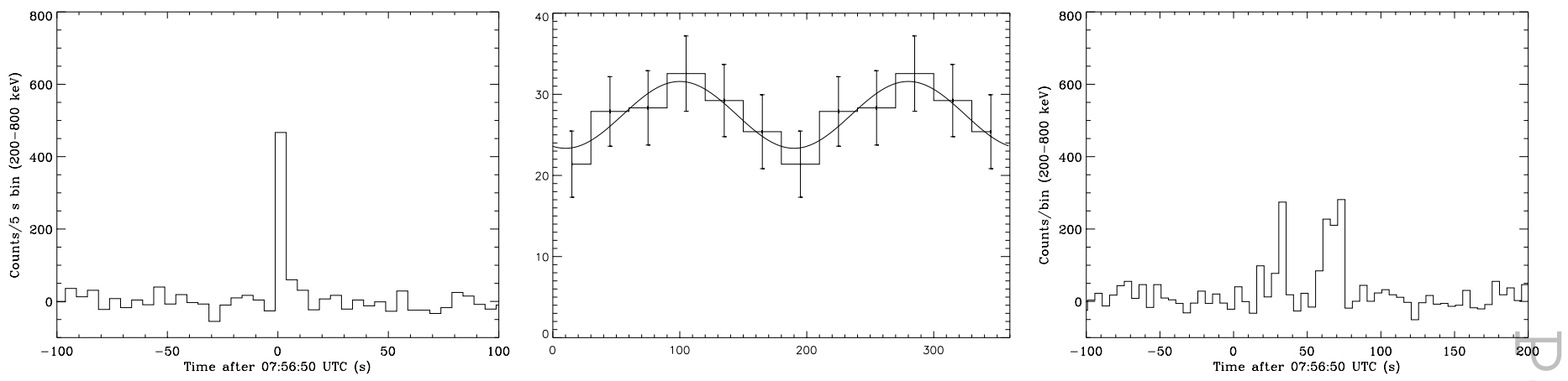

Figure 4: Left: Compton mode light curve of GRB 061122. Center: Polarigram of GRB 061122 in arbitrary flux units. Right: Compton events light curve of GRB 080723B. GRB 061122 has been detected in the Compton imaging mode with a significance of $15 \sigma$ and GRB 080723B of $19 \sigma$.

$[9,8]$.

Nevertheless, due to the low statistics, we currently can not rule out geometrical models, based on peculiar viewing angles (independent from the emission process, synchrotron or inverse Compton) or models based on fragmented fireballs (shotguns, cannon-balls, mini-jets) that are also expected to produce high levels of polarization [14].

\section{Other bursts}

Stimulated by the positive detection of GRB 041219A, we tried to measure the polarization in other bright GRBs detected by IBIS. Here we report our preliminary results. While for GRBs 060901, 070707, 030320, 050525, and 060428C we do not detect any significant signal in the Compton mode ${ }^{1}$, and hence no polarization measurement is possible, for GRB 061122, and GRB 080732B (see Fig. 4), we were able to perform a complete analysis. For the former, integrating over $7 \mathrm{~s}$ of this single peaked GRB, we detect a polarization level of $\Pi=62 \pm 31 \%$, which is compatible with the upper limit of $60 \%$ reported for the same event using the INTEGRAL spectrometer SPI[15]. For the latter we measure a tight upper limit of $10 \%$, but this measure has been made over the entire duration of this double peaked GRB (see Fig. 4). Time resolved analysis of this burst is on-going but the individual peaks may be too weak to detect a significant signal.

\section{Conclusions}

The measurement of polarization is a promising tool for constraining GRB emission models. Despite the moderate sensitivity of the current wide field instrumentation to this kind of signal, sufficiently bright bursts can bring valuable information to a domain - the modeling of the prompt emission - that still needs clarification.

\footnotetext{
${ }^{1}$ As a comparison GRB 041219A was detected with a significance level of $37 \sigma$ over its entire duration.
} 


\section{References}

[1] Gehrels, N., Ramirez-Ruiz, E., \& Fox, D. B., Gamma-Ray Bursts in the Swift Era, ARA\&A 09 (47) 567

[2] Daigne, F., Bosnjak, Z., \& Dubus, G., The origin of the prompt GRB spectrum, in proceedings of 2009 Fermi Symposium, [astro-ph/0912.3743]

[3] Rees, M.J., \& Meszaros, P., Unsteady outflow models for cosmological gamma-ray bursts, ApJ 94 (430) L93

[4] Lyutikov, M., Pariev, V.I., \& Blandford, R.D., Polarization of Prompt Gamma-Ray Burst Emission: Evidence for Electromagnetically Dominated Outflow, ApJ 03 (597) 998

[5] Ubertini, P., Lebrun, F., Di Cocco, G., et al., IBIS: The Imager on-board INTEGRAL, A\&A 03 (411) L131

[6] Forot, M., et al., Polarization of the Crab Pulsar and Nebula as Observed by the INTEGRAL/IBIS Telescope, ApJ 08 (688) L29

[7] Götz, D., et al., Variable Polarization Measured in the Prompt Emission of GRB 041219A Using IBIS on Board INTEGRAL, ApJ 09 (695) L208

[8] Nakar, E., Piran, T. \& Waxman, E., Implications of the bold gamma-ray polarization of GRB 021206, JCAP 03 (01) 005

[9] Granot, J.,Implications of the bold gamma-ray polarization of GRB 021206, ApJ 03 (596) L17

[10] Granot, J. and Königl, A., Linear Polarization in Gamma-Ray Bursts: The Case for an Ordered Magnetic Field, ApJ 03 (596) L83

[11] Spruit, H.C., Daigne, F., \& Drenkhahn, G., Large scale magnetic fields and their dissipation in GRB fireballs, A\&A 01 (369) 694

[12] Kobayashi, S. and Piran, T., \& Sari, R., Can Internal Shocks Produce the Variability in Gamma-Ray Bursts?, ApJ 97 (490) 92

[13] Daigne, F., \& Mochkovitch, R., Gamma-ray bursts from internal shocks in a relativistic wind: temporal and spectral properties, MNRAS 98 (296) 275

[14] Lazzati D., \& Begelman, M.C., Polarization Signature of Gamma-Ray Bursts from Fragmented Fireballs, ApJ 09 (700) L141

[15] McGlynn, S., et al., High energy emission and polarisation limits for the INTEGRAL burst GRB $061122, A \& A 09$ (499) 465 